\title{
Atomically Thin Heterostructures based on Single- Layer Tungsten Diselenide and Graphene
}

Yu-Chuan Lin, ${ }^{1}$ Chih-Yuan Chang, ${ }^{2}$ Jie Li, ${ }^{3}$ Hui Zhu, ${ }^{4}$ Ram Krishna Ghosh,${ }^{3}$ Rafik Addou, ${ }^{4}$ Bogdan Diaconescu, ${ }^{5}$ Taisuke Ohta, ${ }^{5}$ Xin Peng, ${ }^{4}$ Ning Lu, ${ }^{4}$ Moon J. Kim, ${ }^{4}$ Jeremy T. Robinson, ${ }^{6}$ Robert M, Wallace, ${ }^{4}$ Theresa S. Mayer, ${ }^{3}$ Suman Datta, ${ }^{3}$ Lain-Jong Li, ${ }^{2,7^{*}}$ and Joshua A. Robinson ${ }^{{ }^{*}}$

${ }^{1}$ Department of Materials Science and Engineering and Center for 2-Dimensional and Layered Materials, The Pennsylvania State University, University Park, Pennsylvania, 16802, United States

${ }^{2}$ Institute of Atomic and Molecular Sciences, Academia Sinica, Taipei 10617, Taiwan

${ }^{3}$ Department of Electrical Engineering, The Pennsylvania State University, University Park, Pennsylvania, 16802, United States

${ }^{4}$ Department of Materials Science and Engineering, The University of Texas at Dallas, Richardson, Texas 75080 United States

${ }^{5}$ Sandia National Laboratories, Albuquerque, New Mexico, 87185, Untied States

${ }^{6}$ Naval Research Laboratory, Washington D.C., 20375, United States

${ }^{7}$ Physical Science and Engineering Division, King Abdullah University of Science and Technology, Thuwal, 23955-6900, Saudi Arabia.

Heterogeneous engineering of two-dimensional layered materials, including metallic graphene and semiconducting transition metal dichalcogenides, presents an exciting opportunity to produce highly tunable electronic and optoelectronic systems. In order to engineer pristine layers and their interfaces, epitaxial growth of such heterostructures is desirable. We report the direct growth of highly crystalline, monolayer tungsten diselenide ( $\left.\mathrm{WSe}_{2}\right)$ on epitaxial graphene (EG). Raman spectroscopy, photoluminescence, and scanning tunneling microscopy confirm high-quality $\mathrm{WSe}_{2}$ monolayers; while transmission electron microscopy shows an atomically sharp interface, and low energy electron diffraction confirms near perfect orientation between WSe 2 and EG. Vertical transport measurements across the WSe 2 /EG heterostructure provides evidence that a barrier exists due to the interlayer gap, which is supported by density functional theory that predicts an additional barrier along with $\mathrm{WSe}_{2}$ band offset for transport from $\mathrm{WSe}_{2}$ to Graphene. 
KEYWORDS: Direct growth, heterostructures, graphene, tungsten diselenide (WSe 2 ), LEED/

\section{LEEM, electron tunneling, conductive AFM}

Analogous to the evolution in graphene research ${ }^{1}$ we are at the initial stage of forming and characterizing van der Waals ( $\mathrm{vdW}$ ) heterostructures, where samples are produced mainly through mechanical exfoliation and manual transfer stacking. ${ }^{2}$ Unlike isolated monolayer samples, the transfer stacking process can lead to uncontrollable interface contamination ${ }^{3}$ that in turn results in reduced device performance. ${ }^{2,3}$ Therefore, developing synthetic techniques to form such heterostructures is critical for engineering pristine layers and junction interfaces. Efforts towards this end include the vertical integration of two-dimensional (2D) materials such as molybdenum disulfide $\left(\mathrm{MoS}_{2}\right)$ and hexagonal boron nitride $(\mathrm{hBN})$ on epitaxial graphene (EG). ${ }^{4}$ Similarly, chemical vapor deposited (CVD) graphene grown on $\mathrm{Cu}$ foils has been utilized as "universal template" for the synthesis of vertical hBN or $\operatorname{MoS}_{2},{ }^{5,6}$ or lateral (inplane) hBN/graphene systems. ${ }^{7}$ In either case, monolayer growth control is essential to exploit phenomena such as the direct-gap crossover in transition metal dichalcogenides (TMDs) ${ }^{8}$ or inter-layer coupling that can hybridize the electronic structure of stacked monolayers. ${ }^{9}$ In this report, we demonstrate direct growth of high-quality $\mathrm{WSe}_{2}$ monolayers on epitaxial graphene (EG), and provide evidence that this heterosystem exhibits pristine interfaces, high-quality structural, chemical, and optical properties, and significant tunnel resistances due to the $\mathrm{WSe}_{2} / \mathrm{EG}$ interlayer gap.

Epitaxial graphene on silicon carbide $(\mathrm{SiC})^{10,11}$ is an ideal platform to investigate the nucleation and growth of vdW heterostructures. In particular, EG on SiC eliminates the need for post-growth transfer that is required for chemical vapor deposited graphene and therefore provides a chemically uniform starting surface. Epitaxial graphene is synthesized by Si sublimation from the (0001) plane (Si face) of semi-insulating on-axis $6 \mathrm{H}-\mathrm{SiC}$ (II-VI, Inc.) at $1725{ }^{\circ} \mathrm{C}$, 200 Torr, in ultra-high purity argon (Ar). ${ }^{4} \mathrm{The}$ optimized synthesis leads to uniform step bunching and to an atomically flat graphene surface on the $\mathrm{SiC}$ (0001) plane (Supplemental (Supp.) Fig. S1a, b). Tungsten diselenide ( $\mathrm{WSe}_{2}$ ) layers were subsequently synthesized on EG via vapor phase transport of tungsten tri-oxide $\left(\mathrm{WO}_{3}\right)$ in the presence of $\mathrm{Ar} / \mathrm{H}_{2}$ and selenium vapor at $925-1000{ }^{\circ} \mathrm{C} .{ }^{12}$ Epitaxial graphene plays an important role in the nucleation and growth of $\mathrm{WSe}_{2}$. Raman mapping (Fig. 1a) demonstrates that the $\mathrm{WSe}_{2}\left(\mathrm{~A}_{1 \mathrm{~g}} / \mathrm{E}_{2 \mathrm{~g}}\right.$ peak $\left.\sim 250 \mathrm{~cm}^{-1}\right)$ is present only where the graphene $\left(1580 \mathrm{~cm}^{-1}\right.$ and $\left.2700 \mathrm{~cm}^{-1}\right)$ is present underneath (Fig. 1b). Atomic force microscopy (AFM) indicates that the kinetics of $\mathrm{WSe}_{2}$ nucleation and growth on $\mathrm{EG}$ is slow (see supplemental information), with monolayer coverage remaining $<75 \%$ after a 60 minute exposure to the $\mathrm{WSe}_{2}$ precursors (see supplemental information). As a result, $\mathrm{WSe}_{2}$ domain size is highly dependent on synthesis temperature, with the largest domains being achieved at $1000{ }^{\circ} \mathrm{C}$ (Fig. 1c). The height of 
individual $\mathrm{WSe}_{2}$ domains measures $0.71 \mathrm{~nm}$ (Fig. 1c), and atomic arrangement (Fig. 1d) matches that of $2 \mathrm{H}-\mathrm{WSe}{ }_{2}{ }^{13}$ in good agreement with previous reports of CVD $\mathrm{WSe}_{2}{ }^{12}$

Morphological features in the EG (such as wrinkles, $\mathrm{SiC}$ step edges, and other surface imperfections) appear to directly influence $\mathrm{WSe}_{2}$ monolayer development by acting as a barrier to further lateral growth, or by modifying the registry of the $\mathrm{WSe}_{2}$ layers on epitaxial graphene. A qualitative assessment of the $\mathrm{WSe}_{2}$ in-plane orientation via AFM suggests a narrow distribution with $>80 \%$ of the triangles aligned (+/- 5 degrees) to the underlying graphene (see supplemental information). Using low energy electron microscopy/diffraction (LEEM/LEED) we are able to more precisely quantify the inplane crystalline orientation of $\mathrm{WSe}_{2}$ monolayers on EG (Fig. 1c,e). Unlike the diffuse LEED patterns of monolayer $\mathrm{MoS}_{2}$ or $\mathrm{MoSe}_{2}$ on $\mathrm{SiO}_{2},{ }^{14,15}$ the LEED spots of monolayer WSe$/ \mathrm{EG}$ are sharp (Fig. 1e), resembling that found for twisted bilayer graphene on $\mathrm{SiC} .{ }^{16}$ The red and the blue hexagons in Fig. 1e illustrate the diffraction spots for $\mathrm{WSe}_{2}$ and $\mathrm{EG}$, respectively. The larger $\mathrm{WSe}_{2}$ lattice constant $(3.28 \AA)^{17}$ as compared to graphene $(2.46 \AA)$ means the $\mathrm{WSe}_{2}$ diffraction spots will be closer to the specular beam (central spot), where both crystals display hexagonal symmetry. The ratio of their lattice constants matches the ratio of the hexagons' sizes ( 1.3, extracted from our experiment), demonstrating a $23 \%$ lattice mismatch between $\mathrm{WSe}_{2}$ and graphene. Acquiring diffraction from multiple $\mathrm{WSe}_{2}$ islands simultaneously (Fig. 1e) reveals that the $\mathrm{WSe}_{2}$ is not randomly orientated, but maintains an in-plane orientation mostly aligned to the underlying graphene layer, confirming the aforementioned AFM measurement. A detailed inspection of the sample reveals that diffraction spots of $\mathrm{WSe}_{2}$ show near-perfect alignment with the graphene spots, indicating $+/-5^{\circ}$ variation of the relative orientation of the $\mathrm{WSe}_{2}$ islands with respect to the EG layer (not shown). This distribution of azimuthal orientation is significantly improved compared to the exfoliation samples on $\mathrm{SiO}_{2},{ }^{14}$ and further confirms that the graphene lattice plays an important role in the growth of $\mathrm{WSe}_{2}$. The notable azimuthal alignment between $\mathrm{WSe}_{2}$ and $\mathrm{EG}$ despite a significant lattice mismatch suggests that synthesis of these pristine layers occurs via van der Waals epitaxy. ${ }^{18,19}$

It is well known that the electron reflectivity spectra obtained through LEEM measurements (LEEM-IV) can provide the 'fingerprint' of the EG thickness. ${ }^{20,21}$ By combining the information of EG's thickness together with the density and size of $\mathrm{WSe}_{2}$ islands, we observe a distinct correlation between $\mathrm{WSe}_{2}$ island density and EG thickness: The terraces of $\mathrm{SiC}$ terminated with few layers of EG have a higher density of larger $\mathrm{WSe}_{2}$ islands (Fig. 1e) than ones with thicker EG. The triangles in Fig. 1e are $\mathrm{WSe}_{2}$ islands, and the surrounding EG has a darker grey contrast. The terrace located in the middle of the image has, on average, larger $\mathrm{WSe}_{2}$ islands and higher density. LEEM-IV reveals that this particular terrace is terminated with a monolayer of EG, while the neighboring terraces have bilayer EG. We also note that there are virtually no $\mathrm{WSe}_{2}$ islands on the narrow terraces located between the larger terraces are 
covered by many-layer (3-5 layers) EG. As a result, one must take care to control the layer thickness of the underlying graphene to 1-2 layers since the surface properties and chemical reactivity of many-layer graphene layers precludes the formation of large $\mathrm{WSe}_{2}$ domains, similar to that found for synthesis on graphite. $^{22}$

Synthesis of $\mathrm{WSe}_{2} / \mathrm{EG}$ results in pristine chemical, optical, and structural quality of the heterostructure layers and interfaces. X-ray photoelectron spectroscopy (XPS) confirms there is no measureable reaction between graphene and $\mathrm{WSe}_{2}$ (Fig. 2a, b), and the integrals of high resolution spectra of the Se $3 \mathrm{~d}$ and $\mathrm{W} 4 \mathrm{f}$ peaks leads an estimated Se:W ratio of $\sim 2: 1$. Considering the reported core-level energies of monolayer $\mathrm{WSe}_{2}$ on an insulating sapphire substrate $\left(\mathrm{W}_{4} \mathrm{f}_{7 / 2}\right.$ and $\mathrm{W}_{4} \mathrm{f}_{5 / 2}$ peaks are at $32.8 \mathrm{eV}$ and $35.0 \mathrm{eV}$, respectively; $\mathrm{Se} 3 \mathrm{~d}_{5 / 2}$ and $3 \mathrm{~d}_{3 / 2}$ peaks are at $55.0 \mathrm{eV}$ and $55.9 \mathrm{eV}$, respectively.), ${ }^{12}$ we find the $\mathrm{W}$ and Se peaks are shifted to lower values by approximately $0.1 \mathrm{eV}$. A shift in binding energy towards lower energies indicates the lower electron density in $\mathrm{WSe} / \mathrm{EG}$. In other words, EG withdraws electrons from $\mathrm{WSe}_{2}$ monolayer, leading to p-doped behavior in the $\mathrm{WSe}_{2}$ layer. ${ }^{13}$ This is confirmed via direct measurements of the occupied energy states in XPS, showing that the Fermi level is positioned at $0.72 \mathrm{eV}$, which is $0.11 \mathrm{eV}$ smaller than the intrinsic Fermi level position of intrinsic $1 \mathrm{~L} \mathrm{WSe}_{2}(0.83 \mathrm{eV})$.

Monolayer $\mathrm{WSe}_{2}$ is a direct-gap semiconductor, ${ }^{23}$ which can be confirmed by photoluminescence (PL) measurements (Fig. 2d). To better understand the impact of the graphene underlayer on $\mathrm{WSe}_{2}$, we compare the PL spectra of $\mathrm{WSe}_{2}$ grown on insulating sapphire formed under the same growth conditions. From this comparison three features are apparent: (i) the PL intensity of $\mathrm{WSe}_{2} / \mathrm{EG}$ is quenched by a factor of three (Fig. 2d); (ii) the PL peak position of $\mathrm{WSe} / \mathrm{EG}$ is up-shifted by $35 \mathrm{meV}$ (from $1.625 \mathrm{eV}$ on sapphire to $1.66 \mathrm{eV}$ on EG); and (iii) the full width of half-maximum (FWHM) of the WSe $\mathrm{PL}_{2}$ peak on EG is narrower than $\mathrm{WSe}_{2}$-on-sapphire ( $38 \mathrm{meV}$ versus $80 \mathrm{meV}$ ). Since the $\mathrm{WSe}_{2}$ crystalline quality is known to be high on EG (based on LEED, STM, and Raman, Fig. 1), the PL quenching is likely a result of photo-generated charge carriers transferring from $\mathrm{WSe}_{2}$ to EG. ${ }^{24,25}$ Zhang et al. ${ }^{24}$ proposed the observed quenching in $\mathrm{MoS}_{2} / \mathrm{Gr}$ is due to the exciton splitting by the built-in electrical field between 1L CVD graphene and $1 \mathrm{~L} \mathrm{CVD} \mathrm{MoS}_{2}$. Additionally, Shim et al. ${ }^{25}$ observed quenching in $\mathrm{MoSe}_{2} /$ graphene heterostructures due to the faster non-radiative recombination process. It is likely that the quenching process for $\mathrm{WSe}_{2} / \mathrm{EG}$ observed here is similar in nature, especially since there is p-type doping in $\mathrm{WSe}_{2}$ when in proximity to EG. The second feature (red shift of PL peak) may be the result of strain, doping, or defects. ${ }^{25}$ Within the resolution of our measurements (LEED, STM, and PL), we find the WSe 2 defect density to be low; however, there is measureable doping of the $\mathrm{WSe}_{2}$ as a result of the EG underlayer (based on XPS). Doping is known to shift PL signatures in $\mathrm{MoS}_{2},{ }^{26}$ and we believe is the primary contributor to the measured PL shift observed in this work. Finally, the PL properties of $\mathrm{WSe}_{2}$ on an insulating substrate $\left(\mathrm{Al}_{2} \mathrm{O}_{3}\right.$; Fig 2c) versus on graphene is very similar to a recent report comparing in 
$\mathrm{MoS}_{2} / \mathrm{SiO}_{2}$ versus $\mathrm{MoS}_{2} / \mathrm{EG}$ and $\mathrm{MoS}_{2} / \mathrm{hBN}^{27}$ The narrower peak width suggests that the interface between the $\mathrm{WSe}_{2}$ and $\mathrm{EG}$ is pristine, with no dangling bonds contributing to interface roughness or surface optical phonon scattering that in turn leads to an improved optoelectronic quality.

Cross-sectional TEM reveals that the uniformity and structure of the underlying graphene can significantly impact the nucleation, growth, and quality of the $\mathrm{WSe}_{2}$ overlayer. Wherever pristine graphene is present, the $\mathrm{WSe}_{2}$ overlayer is highly crystalline, with no observable defects (Fig. 2e). Additionally, the EG interlayer distance is measured to be $3.64 \AA$ (the typically observed graphene layer spacing in $\mathrm{EG} / \mathrm{SiC}$ ), ${ }^{28}$ while the $\mathrm{WSe}_{2} / \mathrm{EG}$ layer spacing is $5.23 \AA$, with a $\mathrm{WSe}_{2}$ thickness of $6.45 \AA$ (see supplemental information). This is consistent with $6.49 \AA$ for monolayer $\mathrm{WSe}_{2}{ }^{17}$ In the case where the graphene underlayer is defective, we observe increased disorder in the $\mathrm{WSe}_{2}$ (Fig. 2f). We note that unlike the $\mathrm{MoS}_{2}$ grown on $\mathrm{EG},{ }^{4}$ in which the $\mathrm{SiC}(1 \overline{1} 0 \mathrm{n})$ step edges and $\mathrm{EG}$ wrinkles serve as nucleation sites for $\mathrm{MoS}_{2}$ growth, the $\mathrm{WSe}_{2}$ abruptly stops at the edge of the (1) $\left.10 \mathrm{n}\right)$ plane, preferring to grow only on EG synthesized on the $\mathrm{SiC}$ (0001) plane. It is also useful to note that $\mathrm{WSe}_{2}$ is sensitive to damage during TEM investigations and it is not stable under high-energy electrons (also seen in LEEM at high electron beam intensity).

Vertical diode structures (Fig. 3a,b) and conductive atomic force microscopy (C-AFM; Fig. 3c,d) provide a direct means to probe the nanoscale electrical properties of $\mathrm{WSe}_{2} /$ graphene heterostructures, and assists in identifying the utility of these materials for advanced electronic and optoelectronic architectures. Comparing AFM surface topography and conductivity acquired at $\mathrm{V}_{\text {bias }}=0.1 \mathrm{~V}$ (Fig. $3 \mathrm{c}$ and 3d) clearly indicates that a barrier to transport exists in the heterojunction regions. The mapping also reveals that the $\mathrm{WSe}_{2}$ is uniformly resistive, while low resistance contact is possible on the graphene layer, with EG wrinkles and $\mathrm{SiC}$ step edges exhibiting enhanced conduction through the AFM tip. Individual current-voltage (I-V) measurements in C-AFM, and on the diode structures confirm the presence of a tunnel barrier to vertical transport with turn-on occurring at $> \pm 1.5 \mathrm{~V}$ (Fig. $3 \mathrm{e}$ and inset). Prior to turn-on, the vertical heterostructure exhibits a resistance of $\sim 4.5 \times 10^{10} \mathrm{Ohms}$ (45 G $\Omega$ ), five orders of magnitude higher than the measured Pd/EG resistance $\left(\sim 1.4 \times 10^{5}\right.$ Ohms), suggesting that the interlayer gap at the $\mathrm{WSe}_{2} / \mathrm{EG}$ interface, as well as the band mis-alignment of $\mathrm{WSe}_{2} / \mathrm{EG}$ leads to a tunnel resistance $\sim 3 \times 10^{5}$ Ohms. Beyond the turn-on voltage, the resistance decreases to $2.3 \times 10^{6}$ Ohms (Fig. 3e and supplemental), indicating there is still significant resistance to current flow (>20x higher than Pd/EG). The non-symmetric I-V curve demonstrates that the barrier height across the $\mathrm{WSe}_{2} / \mathrm{EG}$ gap is not symmetric - carriers have a lower barrier from the $\mathrm{WSe}_{2}$ film to the graphene. The C-AFM measurement on $\mathrm{WSe}_{2} / \mathrm{EG}$ also provides very similar I-V results (Fig. 3e, inset, blue curve). Meanwhile, the hysteresis is greater at negative bias, possibly due to the induced charge impurities at the interface between $\mathrm{H}_{2}$ - 
passivated $\mathrm{SiC}$ and $\mathrm{EG},{ }^{29}$ while positive bias exhibits nearly hysteresis-free transport (see supplemental information).

To realize the role of interlayer gap between $\mathrm{WSe} / \mathrm{EG}$ we have also calculated the effective potential (i.e. the potential due to the interaction of an electron with other electrons) of a $\mathrm{WSe}_{2} / \mathrm{graphene}$ heterostructure as a function of position through the vertical structure (Fig. 4a) via density functional theory (DFT) (see Supplemental). As there is no dangling bonds in selenium (within $\mathrm{WSe}_{2}$ ) and carbon atoms so this analysis shows that the electron 'feels' a finite potential barrier to vertical transport due to the interlayer gap between $\mathrm{WSe}_{2}$ and $\mathrm{EG}^{30}$ To identify if this effective potential barrier leads to a true transport barrier, we have also performed non-equilibrium Green's function (NEGF) calculation in a $\mathrm{Pd} / \mathrm{WSe}_{2} / \mathrm{EG}$ device (Fig $3 \mathrm{a}, \mathrm{b}$ and $4 \mathrm{~b}$ ) to get the space (i.e. real space) resolved local density of states (LDOS) which is shown in Fig. 4c. This plot depicts the portion of LDOS from $\mathrm{WSe}_{2}$ to first layer of EG ( $<29 \AA$ defines Pd and > 35 $\AA$ defines the higher layer of EG to develop the electrodes). It shows that within the interlayer gap the LDOS is absent and this describes that the interlayer gap is effectively acting as an extra barrier over $\mathrm{WSe}_{2}$ conduction band offset. It can also be seen from the projected contour line of LDOS (Fig 4d) which expresses the boundary from zero LDOS to relatively high LDOS values of this LDOS contour plot that it gives a barrier height of about $1.85 \mathrm{eV}$ relative to the Fermi level (Fig 4d, inset) of the device and this acts as an barrier for thermionic emission of electrons in this device. This model utilizes intrinsic $\mathrm{WSe}_{2}$ with pristine contacts; however, it provides strong evidence that the interlayer plays a critical role in the measured resistance of the layer stack. In our model, the barrier due to the interface gap mainly depends upon the interface gap thickness as well as the interaction among different atoms of the different layers. With the decrease of interface gap, the interaction (especially columbic interaction) increases and it reduces the barrier height. Structure optimization in the DFT simulations indicates that the $\mathrm{Pd} / \mathrm{WSe}_{2}$ interface gap is lower than the $\mathrm{WSe}_{2} /$ graphene and thus the barrier height is predicted to be significantly less than $\mathrm{WSe}_{2} / \mathrm{EG}$. As a result, the electron experiences the maximum thermionic barrier height at the $\mathrm{WSe}_{2}$ /graphene interface. However, we also note that transport barriers are not insignificant at the metal/WSe ${ }_{2}$ contact interface, where Schottky barrier heights of $\sim 0.95 \mathrm{eV}$ are possible. $^{31}$

In conclusion, we demonstrate a synthetic route to forming $\mathrm{WSe}_{2} /$ epitaxial graphene heterostructures via van der Waals epitaxy. Even though the lattice mismatch between $\mathrm{WSe}_{2}$ and graphene is shown to be $23 \%$, the heterostructure is highly commensurate, indicating the potential to grow single crystal heterostructures over large areas. Additionally, we provide evidence that the structural, chemical, and optical properties of the $\mathrm{WSe}_{2}$ grown on graphene match or exceed that of mechanically exfoliated $\mathrm{WSe}_{2}$ films. Finally, $\mathrm{WSe}_{2} / \mathrm{EG}$ diode structures and C-AFM indicate that efficient tunneling is possible 
through the $\mathrm{WSe}_{2}$ layer to graphene, and the primary source of tunneling resistance occurs at the interlayer gap between the $\mathrm{WSe}_{2}$ and graphene layer.

\section{ASSOCIATED CONTENT}

\section{Supporting Information.}

Synthesis, device fabrication, and modelling procedures. AFM, STM, PL, and Raman of epitaxial graphene and $\mathrm{WSe}_{2}$ /graphene. This material is available free of charge via the Internet at http://pubs.acs.org.

\section{AUTHOR INFORMATION}

\section{Corresponding Author:}

*jrobinson@psu.edu; *lance.sinica@gmail.com

\section{ACKNOWLEDGMENT}

Y.-C. Lin and C.-Y. Chang contributed equally to this work. This work at Penn State and UT Dallas was supported by the Center for Low Energy Systems Technology (LEAST), one of six centers supported by the STARnet phase of the Focus Center Research Program (FCRP), a Semiconductor Research Corporation program sponsored by MARCO and DARPA. Device fabrication was supported by the Pennsylvania State University Materials Research Institute Nanofabrication Lab and the National Science Foundation Cooperative Agreement No. ECS0335765. The work at SNL was supported by the US DOE Office of Basic Energy Sciences (BES), Division of Materials Science and Engineering and by Sandia LDRD. Sandia National Laboratories is a multi-program laboratory managed and operated by Sandia Corporation, a wholly owned subsidiary of Lockheed Martin Corporation, for the U.S. Department of Energy's National Nuclear Security Administration under contract DE-AC04-94AL85000. LJL acknowledges the support from Academia Sinica Taiwan, KAUST Saudi Arabia, and US AFOSR-BRI. 


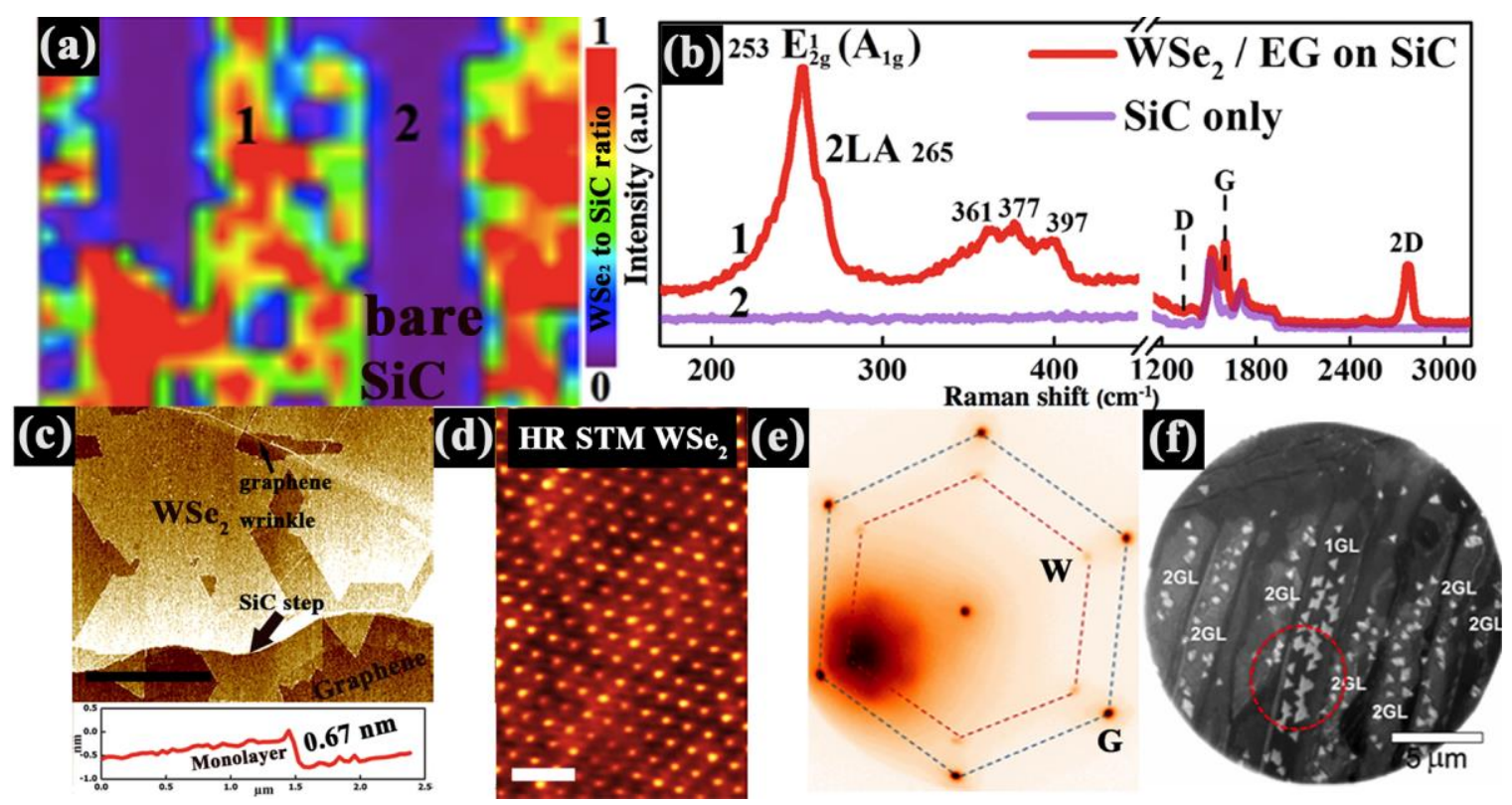

Figure 1: Raman spectroscopy $(\lambda=488 \mathrm{~nm})$ reveals that $(a, b)$ WSe2 monolayers selectively growing on EG and not on bare SiC. Atomic force microscopy (c) confirms WSe 2 domains are 3-5 $\mu \mathrm{m}$ along an edge, and with coverage of $>50 \%$ after a 30 minute growth (scale bar is $5 \mu \mathrm{m})$. High resolution STM (d) also demonstrates a high quality atomic structure (0.35 V, $1.5 \mathrm{nA}$, scale bar is $1 \mathrm{~nm}$ ) and confirms the hexagonal pattern characteristic of $2 \mathrm{H}$-WSe $\mathrm{e}_{2}$ LEED patterns (e) of the WSe 2 (W) on EG (G) confirms a close azimuthal alignment of WSe 2 and EG lattices, and LEEM imaging (f) illustrates the impact of graphene layer thickness on WSe 2 domain formation, where 1-2 layers of EG yield ideal surfaces for large domain WSe2. 

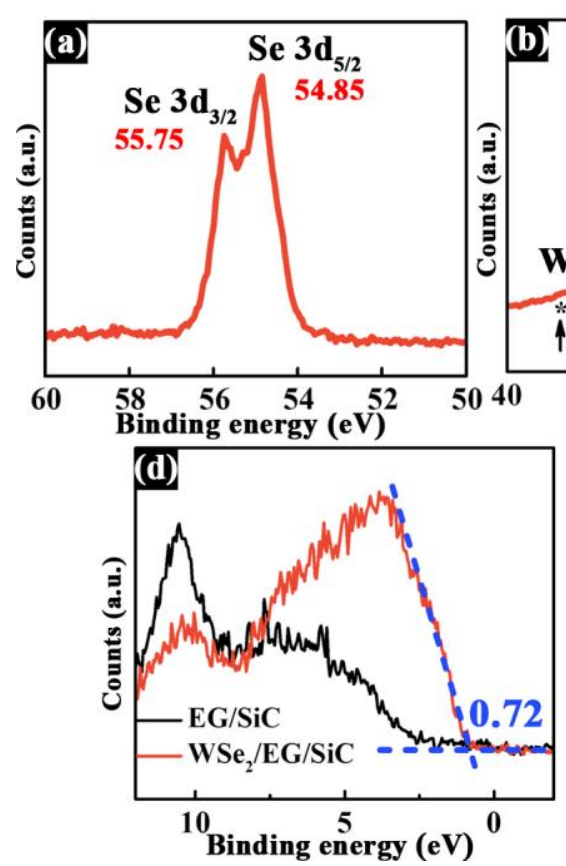

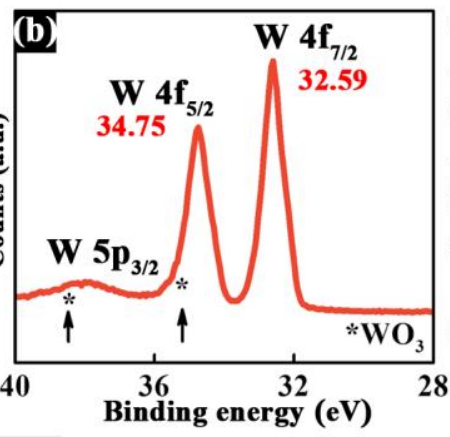

(e)

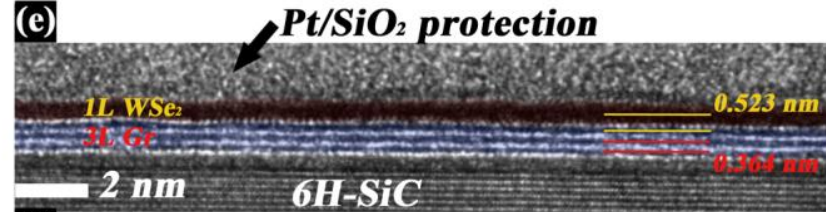

(f)

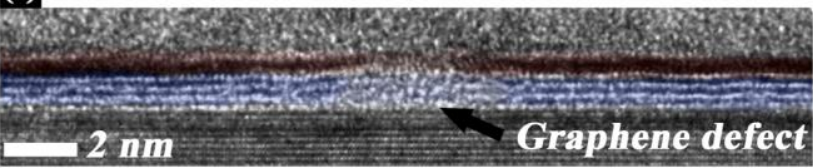

Figure 2: X-ray photoelectron spectroscopy $(a, b, c)$ of the WSe $/$ EG heterostructure reveals that the only bonding within the heterostructure is tungsten to selenium. The position of the individual binding energies of W $4 \mathrm{f}$ (a) and Se 3d (b) are shifted $\sim 0.1 \mathrm{eV}$ below that of CVD WSe2 grown on sapphire, ${ }^{12}$ indicating p-type doping of the WSe $2 .{ }^{13}$ This is confirmed via (d) valance band maxima (VBM) measurements of the WSe 2 /EG/SiC system, which is measured to be $0.72 \mathrm{eV}$ below

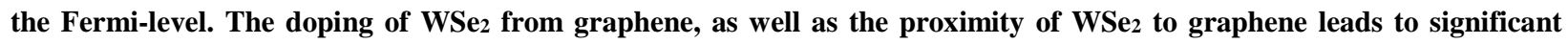
modifications to the photoluminescence (c) of monolayer WSe $2 / \mathrm{EG}$ compared to WSe $/$ /sapphire. Transmission electron microscopy (e,f) demonstrates that pristine interlayer gaps are possible when the underlying EG is defect free (e), however, defects in the graphene are translated to the WSe2 overlayers very effectively (f), resulting in a degraded heterostructure. 

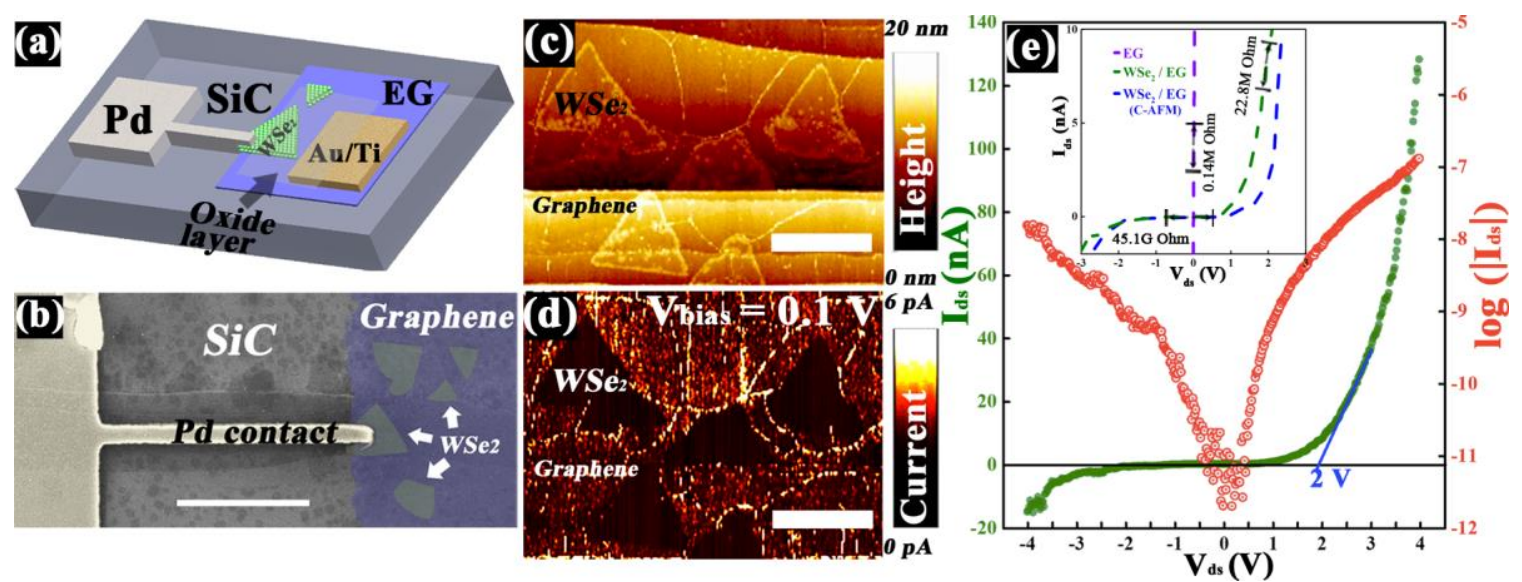

Figure 3: Nanoscale current-voltage characterization indicates that the interlayer gap plays a strong role in vertical transport resistance. The WSe $/$ EG diode structure (a,b) consists of a Pd contact to $\mathrm{WSe}_{2}$, Ti/Au contact to EG, and an oxide over layer to passivate the WSe2 surface. The device performance was compared to conductive AFM (platinum tip) as a means to identify how nanoscale transport impacts mesoscale transport in the device structure. Atomic force microscopy indicates that the topography (c) is close correlated with measure tunneling current (d) between the AFM tip and EG. The measured current versus voltage (I-V) curves from WSe 2 /EG diodes and bare EG (e) and C-AFM measurement on WSe 2 EG (e, inset) confirm a large barrier to transport through the heterostructure $\left(\sim 3 \times 10^{5}\right.$ Ohms $)$ as well as $\sim 10^{5}$ on/off ratio and turn-on voltage of $\sim 2 \mathrm{~V}$. (b, c, and d scale bar: $\left.1 \mu \mathrm{m}\right)$ 

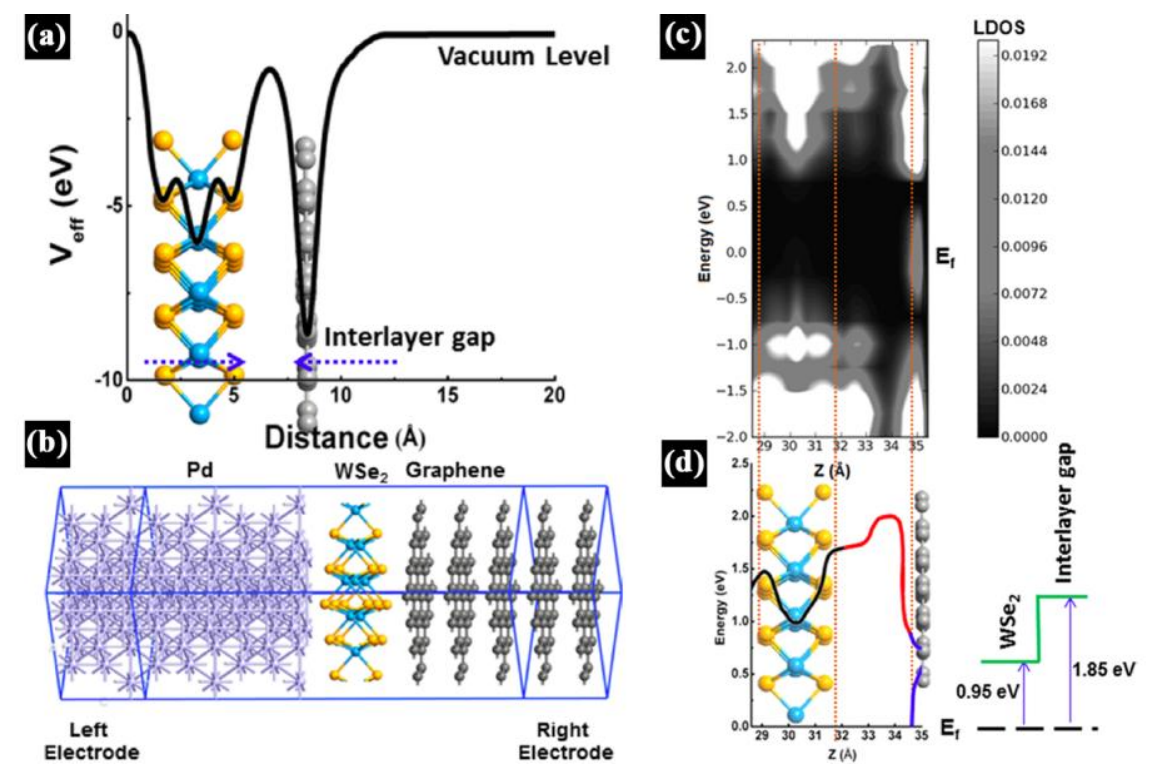

Figure 4: The effective potential of a pristine WSe 2 /EG heterostructure supercell (a) calculated by density functional theory (DFT) along the out of plane direction demonstrates that a significant finite barrier to electron transport can exist as a result of the interlayer gap, (b), atomistic view of the Pd-WSe2-EG two terminal device for vertical transport. (c) Corresponding local density of states (LDOS) within the range between WSe 2 and first layer of EG of this device and (d) projected contour line of LDOS which expresses the boundary that defines the barrier height (zero LDOS to relatively high LDOS values).

\section{REFERENCES}

(1) Geim, A. K.; Grigorieva, I. V. Nature 2013, 499, 419-425.

(2) Britnell, L.; Gorbachev, R. V; Jalil, R.; Belle, B. D.; Schedin, F.; Mishchenko, A.; Georgiou, T.; Katsnelson, M. I.; Eaves, L.; Morozov, S. V; Peres, N. M. R.; Leist, J.; Geim, A. K.; Novoselov, K. S.; Ponomarenko, L. A. Science 2012, 335, 947-950.

(3) Haigh, S. J.; Gholinia, A.; Jalil, R.; Romani, S.; Britnell, L.; Elias, D. C.; Novoselov, K. S.; Ponomarenko, L. A.; Geim, A. K.; Gorbachev, R. Nat. Mater. 2012, 11, 764-767.

(4) Lin, Y.-C.; Lu, N.; Perea-Lopez, N.; Li, J.; Lin, Z.; Peng, X.; Lee, C. H.; Sun, C.; Calderin, L.; Browning, P. N.; Bresnehan, M. S.; Kim, M. J.; Mayer, T. S.; Terrones, M.; Robinson, J. A. ACS Nano 2014, 8, 3715-3723.

(5) Liu, Z.; Song, L.; Zhao, S.; Huang, J.; Ma, L.; Zhang, J.; Lou, J.; Ajayan, P. M. Nano Lett. 2011, 11, 2032-2037.

(6) Shi, Y.; Zhou, W.; Lu, A.-Y.; Fang, W.; Lee, Y.-H.; Hsu, A. L.; Kim, S. M.; Kim, K. K.; Yang, H. Y.; Li, L.-J.; Idrobo, J.-C.; Kong, J. Nano Lett. 2012, 12, 2784-2791. 
(7) Levendorf, M. P.; Kim, C.-J.; Brown, L.; Huang, P. Y.; Havener, R. W.; Muller, D. A.; Park, J. Nature 2012, 488, 627-632.

(8) Ohta, T.; Robinson, J. T.; Feibelman, P. J.; Bostwick, A.; Rotenberg, E.; Beechem, T. E. Phys. Rev. Lett. 2012, 109, 186807.

(9) Robinson, J. T.; Schmucker, S. W.; Diaconescu, C. B.; Long, J. P.; Culbertson, J. C.; Ohta, T.; Friedman, A. L.; Beechem, T. E. ACS Nano 2013, 7, 637-644.

(10) De Heer, W. A.; Berger, C.; Wu, X.; First, P. N.; Conrad, E. H.; Li, X.; Li, T.; Sprinkle, M.; Hass, J.; Sadowski, M. L.; Potemski, M.; Martinez, G. Solid State Commun. 2007, 143, 92-100.

(11) Robinson, J.; Weng, X.; Trumbull, K.; Cavalero, R.; Wetherington, M.; Frantz, E.; Labella, M.; Hughes, Z.; Fanton, M.; Snyder, D. ACS Nano 2010, 4, 153-158.

(12) Huang, J.-K.; Pu, J.; Hsu, C.-L.; Chiu, M.-H.; Juang, Z.-Y.; Chang, Y.-H.; Chang, W.-H.; Iwasa, Y.; Takenobu, T.; Li, L.-J. ACS Nano 2013, 1.

(13) McDonnell, S.; Azcatl, A.; Addou, R.; Gong, C.; Battaglia, C.; Chuang, S.; Cho, K.; Javey, A.; Wallace, R. M. ACS Nano 2014, 8, 6265-6272.

(14) Yeh, P.-C.; Jin, W.; Zaki, N.; Zhang, D.; Sadowski, J. T.; Al-Mahboob, A.; van der Zande, A. M.; Chenet, D. A.; Dadap, J. I.; Herman, I. P.; Sutter, P.; Hone, J.; Osgood, R. M. Phys. Rev. B 2014, 89, 155408.

(15) Zhang, Y.; Chang, T.-R.; Zhou, B.; Cui, Y.-T.; Yan, H.; Liu, Z.; Schmitt, F.; Lee, J.; Moore, R.; Chen, Y.; Lin, H.; Jeng, H.-T.; Mo, S.-K.; Hussain, Z.; Bansil, A.; Shen, Z.-X. Nat. Nanotechnol. 2014, 9, 111-115.

(16) Ohta, T.; Beechem, T. E.; Robinson, J. T.; Kellogg, G. L. Phys. Rev. B 2012, 85, 075415.

(17) Wilson, J. A.; Yoffe, A. D. Adv. Phys. 1969, 18, 193-335.

(18) Koma, A. Thin Solid Films 1992, 216, 72-76.

(19) Ji, Q.; Zhang, Y.; Gao, T.; Zhang, Y.; Ma, D.; Liu, M.; Chen, Y.; Qiao, X.; Tan, P.-H.; Kan, M.; Feng, J.; Sun, Q.; Liu, Z. Nano Lett. 2013, 13, 3870-3877.

(20) Hibino, H.; Kageshima, H.; Maeda, F.; Nagase, M.; Kobayashi, Y.; Yamaguchi, H. Phys. Rev. B 2008, 77, 075413.

(21) Riedl, C.; Coletti, C.; Iwasaki, T.; Zakharov, A. A.; Starke, U. Phys. Rev. Lett. 2009, 103, 246804.

(22) Zhang, C.; Johnson, A.; Hsu, C.-L.; Li, L.-J.; Shih, C.-K. Nano Lett. 2014, 14, 2443-2447. 
(23) Terrones, H.; Del Corro, E.; Feng, S.; Poumirol, J. M.; Rhodes, D.; Smirnov, D.; Pradhan, N. R.; Lin, Z.; Nguyen, M. A. T.; Elías, A. L.; Mallouk, T. E.; Balicas, L.; Pimenta, M. A.; Terrones, M. Sci. Rep. 2014, 4, 4215.

(24) Zhang, W.; Chuu, C.-P.; Huang, J.-K.; Chen, C.-H.; Tsai, M.-L.; Chang, Y.-H.; Liang, C.T.; Chen, Y.-Z.; Chueh, Y.-L.; He, J.-H.; Chou, M.-Y.; Li, L.-J. Sci. Rep. 2014, 4, 3826.

(25) Shim, G. W.; Yoo, K.; Seo, S.-B.; Shin, J.; Jung, D. Y.; Kang, I.-S.; Ahn, C. W.; Cho, B. J.; Choi, S.-Y. ACS Nano 2014, 8, 6655-6662.

(26) Mouri, S.; Miyauchi, Y.; Matsuda, K. Nano Lett. 2013, 13, 5944-5948.

(27) Buscema, M.; Steele, G. A.; van der Zant, H. S. J.; Castellanos-Gomez, A. Nano Res. 2014, 7, 561-571.

(28) Weng, X.; Robinson, J. A.; Trumbull, K.; Cavalero, R.; Fanton, M. A.; Snyder, D. Appl. Phys. Lett. 2010, 97, 201905.

(29) Ristein, J.; Mammadov, S.; Seyller, T. Phys. Rev. Lett. 2012, 108, 246104.

(30) Xie, X.; Sarkar, D.; Liu, W.; Kang, J.; Marinov, O.; Jamal Deen, M.; Banerjee, K. ACS Nano 2014, 8, 5633-5640.

(31) Das, S.; Appenzeller, J. Appl. Phys. Lett. 2013, 103, 103501. 